\title{
Generalized Synchronization of Lorenz Chaotic System with Star Network
}

\author{
Mohammad Ali Khan \\ Department of Mathematics, Garhbeta Ramsundar Vidyabhaban, \\ West Bengal, India \\ E-mail: mdmaths@gmail.com
}

\begin{abstract}
In this paper, we propose the theory for generalized synchronization (GS) of a chaotic star network. We derive sufficient conditions for generalized synchronization of any chaotic system on a star network. The relationship among the state variables at GS are completely known in our method. The effectiveness and feasibility of the synchronization strategy is confirmed and demonstrated by numerical simulation.
\end{abstract}

Keywords: Generalized synchronization (GS), Lorenz chaotic system, Star network.

\section{Introduction}

The study of synchronization in coupled chaotic system is of fundamental importance in nature and science [1] due to its potential applications in the field of laser dynamics, electronic circuits, biological systems and secure communication [2]. Synchronization of chaos is a phenomenon that may occur when two or more chaotic oscillators are coupled or when a chaotic oscillator drives another oscillator. If $\left(x_{1}, x_{2}, \ldots, x_{n}\right)$ and $\left(y_{1}, y_{2}, \ldots, y_{n}\right)$ denote the set of dynamical variables that describe the state of the first and second oscillator respectively, then it is said that identical synchronization occurs when there is a set of initial conditions $\left(x_{1}(0), x_{2}(0), \ldots, x_{n}(0)\right)$ and $\left(y_{1}(0), y_{2}(0), \ldots, y_{n}(0)\right)$ such that depending on time $\mathrm{t}$, $\left|x_{i}(t)-y_{i}(t)\right| \rightarrow 0$ for $i=1,2, \ldots n$, when $t \rightarrow \infty$. This type of synchronization is known as identical synchronization [1]. A generalization of this concept was 
proposed by Rulkov et.al. in 1995 [3]. Generalized synchronization occurs when there is a functional $\phi$ such that after a transitory evolution from appropriate initial conditions i.e. $\left(y_{1}(t), y_{2}(t), \ldots, y_{n}(t)\right)=\phi\left(x_{1}(t), x_{2}(t), \ldots, x_{n}(t)\right)$. This means that the dynamical state of one of the oscillator is completely determined by the state of the other. Several other types of synchronization, such as phase synchronization $[4,5,6]$, lag synchronization [7,8,9], adaptive synchronization $[10,11,12,13,14]$, anti-synchronization $[15,16,17,18,19]$ are studied theoretically and observed experimentally. The subject of complex networks not only just possesses a great challenge but also provides a great opportunity for Scientists, Mathematicians, Physicists and Engineers with significant impacts on the modern industry, commercial markets and beyond, therefore is worth further pursuing. The last decade has witnessed the birth of a new movement of interest and research in the study of complex networks. In this context two seminal papers, that by Watts and Strogatz [20] on a small-world networks appeared in Nature in 1998 and that by R. Albert et.al. [21] on scale-free networks appeared one year later in Science. Complex network is a large-scale network whose structure is irregular, complex and dynamically evolving in time. It analyzes the systems with thousands or millions of nodes connected by edges. The nodes in complex network are basic units of a system which has specific power and information content and the edge means the relationship or contact of two basic units.

Recently L.Tang and Z.Kang [22] proposed synchronization scheme for a three dimensional hyperchaotic star network. They have developed identical synchronization among the states of the nodes of a chaotic star network. We discuss our method by taking same hyperchaotic system as L.Tang et.al.[22]. We obtain the results of L.Tang et.al.[22] as a special case of our model. Our synchronization scheme works for any chaotic system. The functional relationship among the state variables of the non-central nodes are known in our synchronization method. We have presented the simulation results to show the efficiency of our method.

\section{Lorenz Chaotic Systems}

We consider the dynamical system

$$
\begin{aligned}
& \dot{x}=\sigma(y-x) \\
& \dot{y}=r x-y-x z \\
& \dot{z}=x y-d z
\end{aligned}
$$

Here $\sigma, \mathrm{r}, \mathrm{d}(>0)$ are parameters. Ed.Lorenz (1963) derives this three-dimensional system from a drastically simplified model of convection rolls in the atmosphere. The same equations also arise in models of lasers and dynamics. This system 
shows a chaotic behavior for $\sigma=10, \mathrm{r}=28$ and $\mathrm{d}=\frac{8}{3}$. The dynamical system (1) can be written as

$\dot{X}=L(X)+N L(X)$

where $L(X)$ is the linear part and $N L(X)$ is the non-linear part of the system. The linear part $L(X)$ can be written as

$L(X)=A X$

where $A$ is a full rank constant matrix and all eigen values have negative real parts. Therefore (2) becomes

$\dot{X}=A X+N L(X)$

where

$$
\dot{X}=\left[\begin{array}{c}
\dot{x} \\
\dot{y} \\
\dot{z}
\end{array}\right], A=\left[\begin{array}{ccc}
-\sigma & -\sigma & 0 \\
r & -1 & 0 \\
0 & 0 & -d
\end{array}\right], X=\left[\begin{array}{c}
x \\
y \\
z
\end{array}\right] \text { and } N L(X)=\left[\begin{array}{c}
2 \sigma y \\
-x z \\
x y
\end{array}\right]
$$

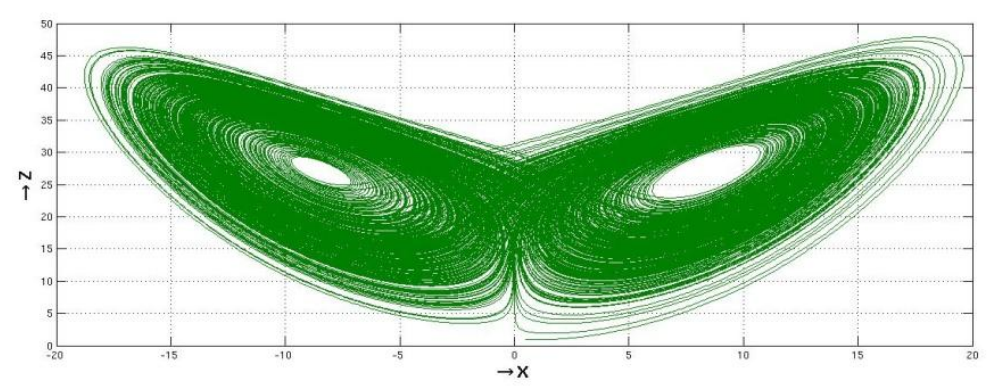

Fig.1: Phase diagram of Lorenz system for $\sigma=10, \mathrm{r}=28$ and $\mathrm{d}=\frac{8}{3}$.

\section{Generalized Synchronization of Star Network}

Star networks are one of the most simple networks topologies. A star network consists of one central node which connects to other nodes. The central node in star network acts as transmit information to other nodes. The star network prevents the passing of the data packets through an excessive number of nodes and it is easy to understand, establish and navigate. The main disadvantage of star network is the high dependence of the system on the functioning of the central node. 


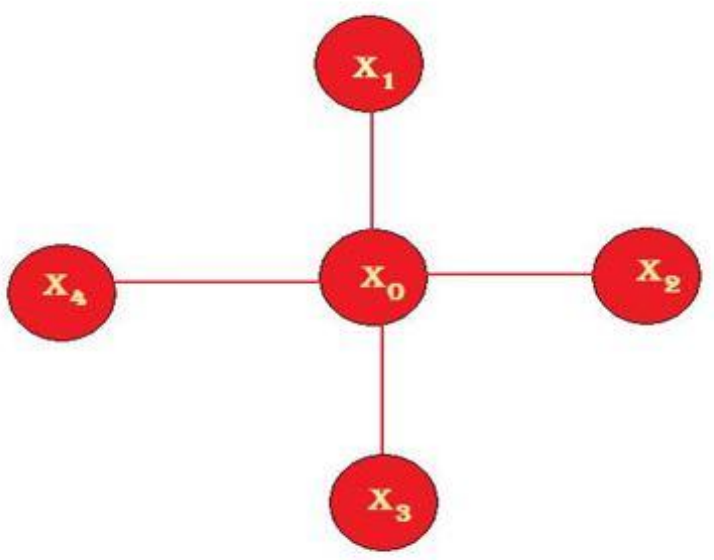

Fig.2. Star network

\section{Theorem 3.1}

A star network with the central node dynamics

$\dot{X}=A X+N L(X)$

where $A X$ is the linear part and $N L(X)$ is the non-linear part of a chaotic system and with the branch nodes dynamics

$\dot{X}_{i}=A X_{i}+\Lambda_{i} N L\left(X_{i}\right)+\mu_{i}\left[N L\left(X_{0}\right)-N L\left(X_{i}\right)\right]$

where $X_{i}(i=1,2,3, \ldots, n)$ are the state vectors of the $i^{\text {th }}$ node of the network and $\mu_{i}(i=1,2,3, \ldots, n)$ are the coefficients of the coupling strength and $\Lambda_{i}(i=1,2,3, \ldots, n)$ are arbitrary matrices such that each $\Lambda_{i}$ commutes with $A$. Then the dynamics of all non-central node will synchronize if and only if $A$ is negative definite matrix and $\Lambda_{i}=\mu_{i}$.

Proof: Let us define the synchronization error as $e_{i}=X_{i}-\Lambda_{i} X_{0}(i=1,2,3, \ldots, n)$. Then the evolutional equation for the synchronization error $e(t)$ is given by

$$
\begin{aligned}
& \dot{e}_{i}=\dot{X}_{i}-\Lambda_{i} \dot{X}_{0} \quad(i=1,2,3, \ldots, n) \\
& =A X_{i}+\Lambda_{i} N L\left(X_{i}\right)+\mu_{i}\left[N L\left(X_{0}\right)-N L\left(X_{i}\right)\right]-\Lambda_{i}\left[A X_{0}+N L\left(X_{0}\right)\right] \\
& =A e_{i}+\left(\Lambda_{i}-\mu_{i}\right)\left[N L\left(X_{i}\right)-N L\left(X_{0}\right)\right]
\end{aligned}
$$


From the above evolution equation we observe that when $\Lambda_{i}=\mu_{i}$, then origin is an asymptotically stable fixed point. Hence the dynamics of the branch node dynamics will synchronize with each other. We notice that the branch node dynamics will not synchronize with the central node dynamics in general. In our model if we choose $\Lambda_{i}=\mu_{i}=I$, then we obtain the results of L.Tang et.al.[22]. In this case the total network will identically synchronize. Since $A$ is invertible matrix , therefore we can have infinite number of choices for matrices $\Lambda_{i}$ which commutes with $A$.

Fig.3. shows the synchronization error tends to zero after a short transient process.

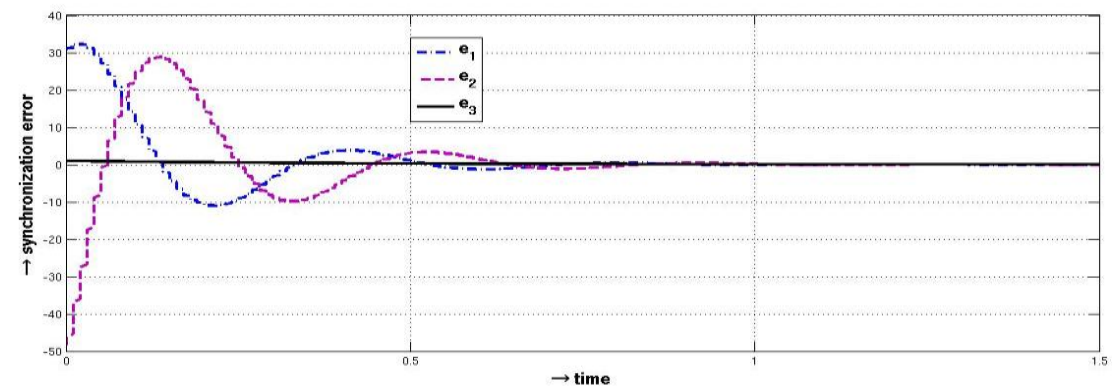

Fig.3: Change of synchronization error over time in star network.

\section{Simulation Results}

We present here four simulation results depending upon different choices of $\Lambda_{i}$ and $\mu_{i}$ and use one central node and three branch nodes. The parameters of the chaotic system are chosen as follows $\sigma=10, r=28$ and $d=\frac{8}{3}$. Fourth order Runge-Kutta method is used for these simulations. We denote the central node state variable by subscript 0 and other node state variables by subscripts 1,2 and 3 . Then the dynamics of star network are governed by following differential equations

$$
\begin{aligned}
& \dot{x}_{0}=\sigma\left(y_{0}-x_{0}\right) \\
& \dot{y}_{0}=-x_{0} z_{0}+r x_{0}-y_{0} \\
& \dot{z}_{0}=-x_{0} y_{0}-d z_{0} \\
& \dot{x}_{i}=-\sigma\left(x_{i}+y_{i}\right)+2 \sigma\left(\lambda_{11}^{i}-\mu_{11}^{i}\right) y_{i}-\left(\lambda_{12}^{i}-\mu_{12}^{i}\right) x_{i} z_{i}+\left(\lambda_{13}^{i}-\mu_{13}^{i}\right) x_{i} y_{i} \\
& +2 \sigma \mu_{11}^{i} y_{0}-\mu_{12}^{i} x_{0} z_{0}+\mu_{13}^{i} x_{0} y_{0} \\
& \dot{y}_{i}=r x_{i}-y_{i}+2 \sigma\left(\lambda_{21}^{i}-\mu_{21}^{i}\right) y_{i}-\left(\lambda_{22}^{i}-\mu_{22}^{i}\right) x_{i} z_{i}+\left(\lambda_{23}^{i}-\mu_{23}^{i}\right) x_{i} y_{i} \\
& +2 \sigma \mu_{21}^{i} y_{0}-\mu_{22}^{i} x_{0} z_{0}+\mu_{23}^{i} x_{0} y_{0}
\end{aligned}
$$


$\dot{z}_{i}=-d z_{i}+2 \sigma\left(\lambda_{31}^{i}-\mu_{31}^{i}\right) y_{i}-\left(\lambda_{32}^{i}-\mu_{32}^{i}\right) x_{i} z_{i}+\left(\lambda_{33}^{i}-\mu_{33}^{i}\right) x_{i} y_{i}$

$+2 \sigma \mu_{31}^{i} y_{0}-\mu_{32}^{i} x_{0} z_{0}+\mu_{33}^{i} x_{0} y_{0}$

$\forall i=1,2,3$

Where

$\Lambda_{i}=\left[\begin{array}{lll}\lambda_{11}^{i} & \lambda_{12}^{i} & \lambda_{13}^{i} \\ \lambda_{21}^{i} & \lambda_{22}^{i} & \lambda_{23}^{i} \\ \lambda_{31}^{i} & \lambda_{32}^{i} & \lambda_{33}^{i}\end{array}\right]$ and $\mu_{i}=\left[\begin{array}{ccc}\mu_{11}^{i} & \mu_{12}^{i} & \mu_{13}^{i} \\ \mu_{21}^{i} & \mu_{22}^{i} & \mu_{23}^{i} \\ \mu_{31}^{i} & \mu_{32}^{i} & \mu_{33}^{i}\end{array}\right]$

\section{Simulation 1}

In this simulation we choose

$$
\begin{aligned}
& \Lambda_{1}=\Lambda_{2}=\Lambda_{3}=\left(\begin{array}{lll}
\lambda & 0 & 0 \\
0 & \lambda & 0 \\
0 & 0 & \lambda
\end{array}\right) \text { with } \lambda \neq 0 \\
& \mu_{1}=\mu_{2}=\mu_{3}=\left(\begin{array}{lll}
\mu & 0 & 0 \\
0 & \mu & 0 \\
0 & 0 & \mu
\end{array}\right) \text { with } \mu \neq 0
\end{aligned}
$$

Then the dynamics of non-central nodes are given by

$$
\begin{aligned}
& \dot{x}_{i}=-\sigma\left(x_{i}+y_{i}\right)+2 \sigma(\lambda-\mu) y_{i}+2 \sigma \mu y_{0} \\
& \dot{y}_{i}=r x_{i}-y_{i}-(\lambda-\mu) x_{i} z_{i}-\mu x_{0} z_{0} \\
& \dot{z}_{i}=-d z_{i}+(\lambda-\mu) x_{i} y_{i}+\mu x_{0} y_{0} \\
& \forall i=1,2,3
\end{aligned}
$$

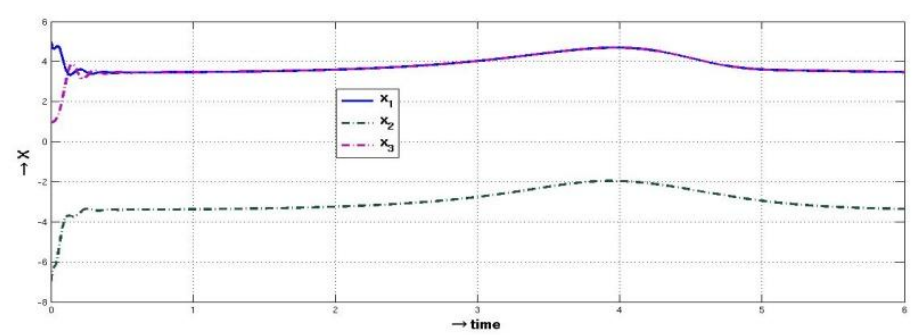

Fig.4(a). Time evolution of $x_{1}, x_{2}$ and $x_{3}$ when $\lambda \neq \mu$ for simulation 1 . 


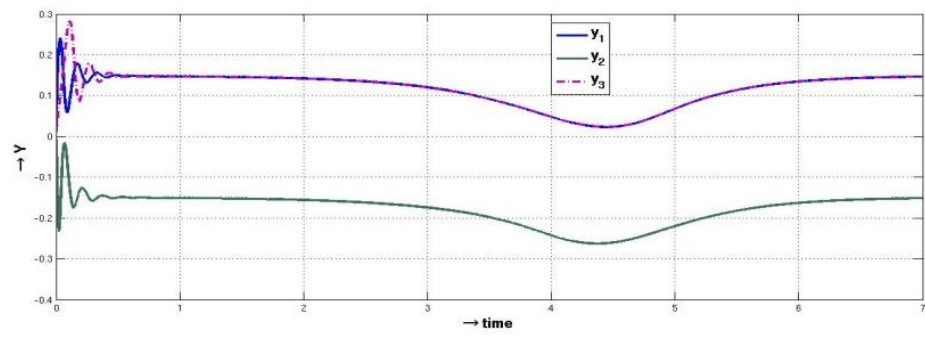

Fig.4(b). Time evolution of $y_{1}, y_{2}$ and $y_{3}$ when $\lambda \neq \mu$ for simulation 1 .

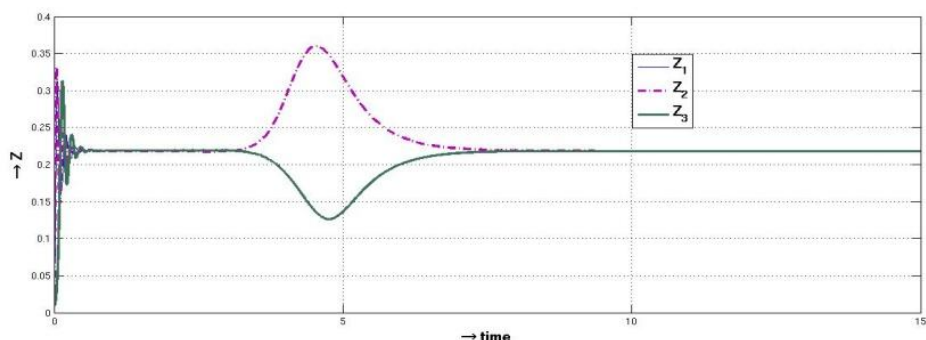

Fig.4(c). Time evolution of $z_{1}, z_{2}$ and $z_{3}$ when $\lambda \neq \mu$ for simulation 1 .

From the Fig.4(a)-Fig.4(c), it is clear that non-central nodes may or may not be synchronize if $\Lambda_{i} \neq \mu_{i},(i=1,2,3)$. But if $\Lambda_{i}=\mu_{i}(i=1,2,3)=\left[\begin{array}{lll}\lambda & 0 & 0 \\ 0 & \lambda & 0 \\ 0 & 0 & \lambda\end{array}\right]$ then the dynamics of non-central nodes are given by

$$
\begin{aligned}
& \dot{x}_{i}=-\sigma\left(x_{i}+y_{i}\right)+2 \sigma \lambda y_{0} \\
& \dot{y}_{i}=r x_{i}-y_{i}-\lambda x_{0} z_{0} \\
& \dot{z}_{i}=-d z_{i}+\lambda x_{0} y_{0} \\
& \forall i=1,2,3
\end{aligned}
$$

Hence by the Theorem 3.1, the non-central nodes are synchronized shown in Fig.5(a)-Fig.5(c).

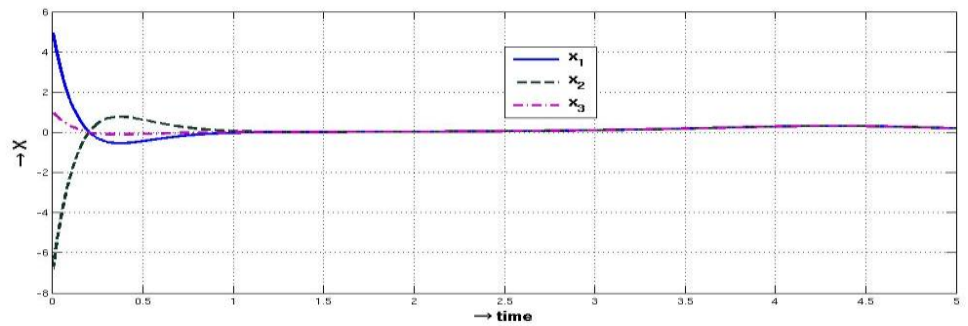

Fig.5(a). Time evolution of $x_{1}, x_{2}$ and $x_{3}$ when $\lambda=\mu$ for simulation 1 . 


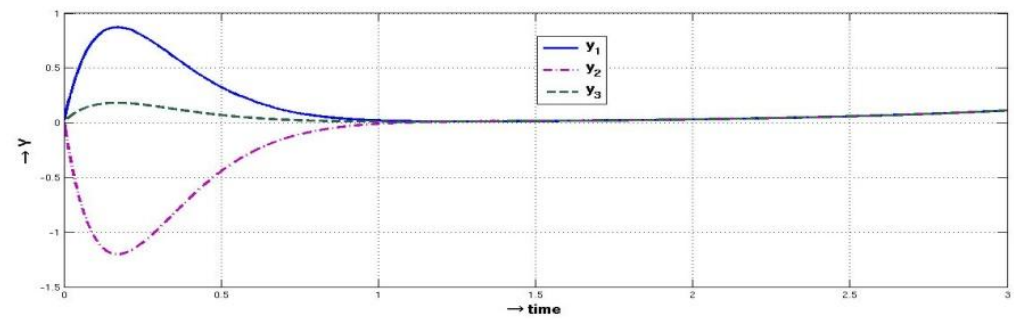

Fig.5(b): Time evolution of $y_{1}, y_{2}$ and $y_{3}$ when $\lambda=\mu$ for simulation 1 .

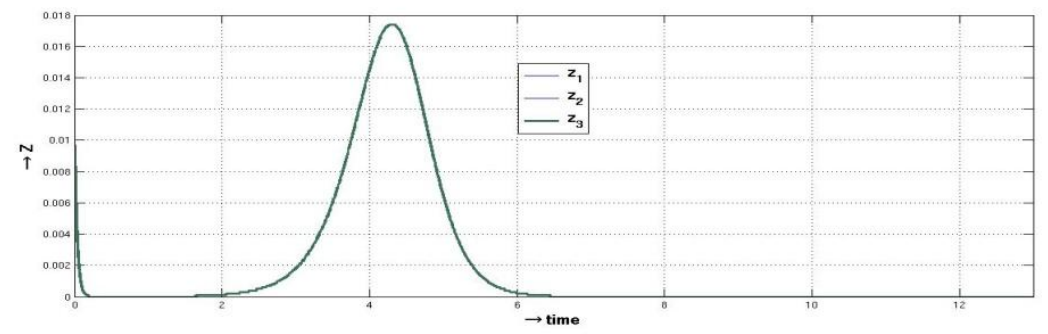

Fig.5(c): Time evolution of $z_{1}, z_{2}$ and $z_{3}$ when $\lambda=\mu$ for simulation 1 .

\section{Simulation 2}

In this simulation we take

$$
\begin{aligned}
& \Lambda_{1}=\Lambda_{2}=\Lambda_{3}=A=\left(\begin{array}{ccc}
-\sigma & -\sigma & 0 \\
r & -1 & 0 \\
0 & 0 & -d
\end{array}\right) \text { and } \\
& \mu_{1}=\mu_{2}=\mu_{3}=A^{-1}=\left(\begin{array}{ccc}
-\frac{1}{\sigma(r+1)} & -\frac{1}{r+1} & 0 \\
-\frac{r}{\sigma(r+1)} & \frac{1}{r+1} & 0 \\
0 & 0 & \frac{1}{d}
\end{array}\right)
\end{aligned}
$$

Then the dynamics of non-central nodes are given by

$$
\begin{aligned}
& \dot{x}_{i}=-\sigma\left(x_{i}+y_{i}\right)+2 \sigma\left(\frac{1}{\sigma(r+1)}-\sigma\right) y_{i}-\left(\frac{1}{r+1}-\sigma\right) x_{i} z_{i}-\frac{2}{r+1} y_{0}+\frac{1}{r+1} x_{0} z_{0} \\
& \dot{y}_{i}=r x_{i}-y_{i}+2 \sigma r\left(1+\frac{1}{\sigma(r+1)}\right) y_{i}+\left(1+\frac{1}{r+1}\right) x_{i} z_{i}-\frac{2 r}{r+1} y_{0}-\frac{1}{r+1} x_{0} z_{0} \\
& \dot{z}_{i}=-d z_{i}-\left(d+\frac{1}{d}\right) x_{i} y_{i}+\frac{1}{d} x_{0} y_{0} \\
& \forall i=1,2,3
\end{aligned}
$$

Here the non-central nodes always not synchronize. But if we choose

$$
\Lambda_{i}=\mu_{i}(i=1,2,3)=A=\left[\begin{array}{ccc}
-\sigma & -\sigma & 0 \\
r & -1 & 0 \\
0 & 0 & -d
\end{array}\right]
$$


Then the dynamics of non-central nodes are given by

$$
\begin{aligned}
& \dot{x}_{i}=-\sigma\left(x_{i}+y_{i}\right)-2 \sigma^{2} y_{0}+\sigma x_{0} z_{0} \\
& \dot{y}_{i}=r x_{i}-y_{i}+2 \sigma r y_{0}-x_{0} z_{0} \\
& \dot{z}_{i}=-d z_{i}-d x_{0} y_{0} \\
& \forall i=1,2,3
\end{aligned}
$$

According to Theorem 3.1. it is clear that non-central nodes are synchronized shown in Fig.6(a)-Fig.6(c).

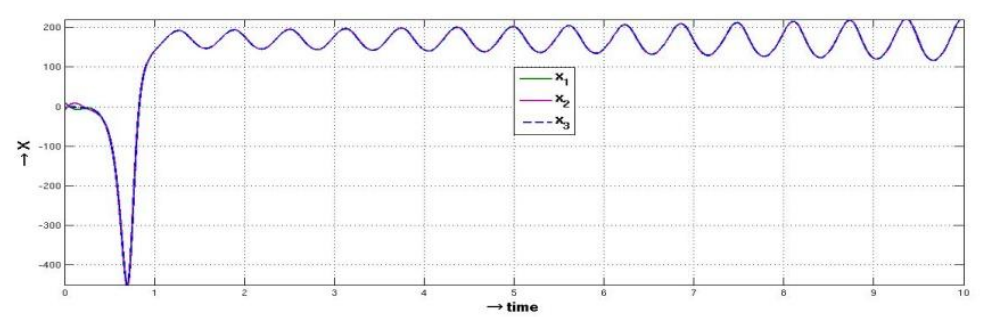

Fig.6(a): Time evolution of $x_{1}, x_{2}$ and $x_{3}$ when $\lambda=\mu$ for simulation 2 .

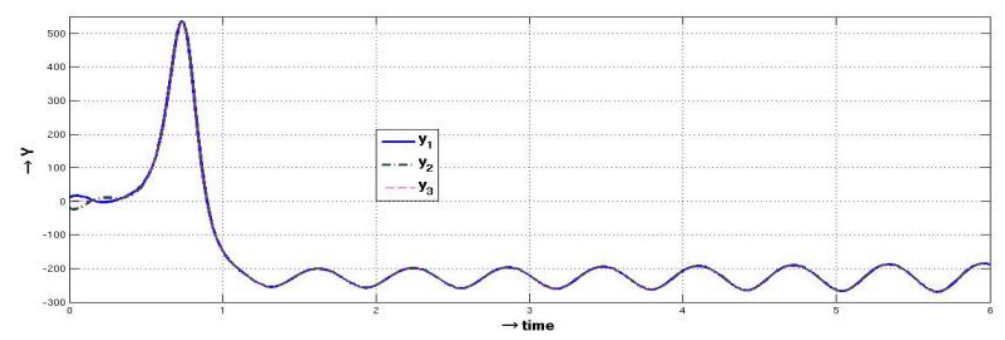

Fig.6(b): Time evolution of $y_{1}, y_{2}$ and $y_{3}$ when $\lambda=\mu$ for simulation 2 .

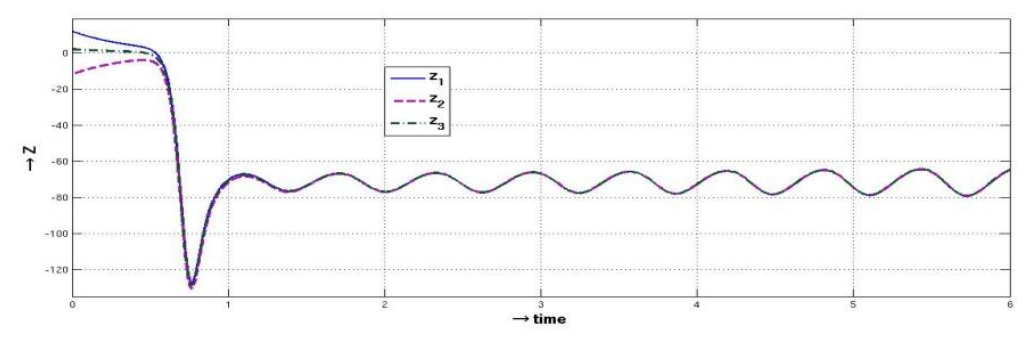

Fig.6(c): Time evolution of $z_{1}, z_{2}$ and $z_{3}$ when $\lambda=\mu$ for simulation 2 .

\section{Simulation 3}

In this simulation we take 
$\Lambda_{1}=\Lambda_{2}=\Lambda_{3}=A^{-1}=\left(\begin{array}{ccc}-\frac{1}{\sigma(r+1)} & -\frac{1}{r+1} & 0 \\ -\frac{r}{\sigma(r+1)} & \frac{1}{r+1} & 0 \\ 0 & 0 & \frac{1}{d}\end{array}\right)$

and

$\mu_{1}=\mu_{2}=\mu_{3}=A=\left(\begin{array}{ccc}-\sigma & -\sigma & 0 \\ r & -1 & 0 \\ 0 & 0 & -d\end{array}\right)$

Then the dynamics of non-central nodes are given by

$$
\begin{aligned}
& \dot{x}_{i}=-\sigma\left(x_{i}+y_{i}\right)+2 \sigma\left(\sigma-\frac{1}{\sigma(r+1)}\right) y_{i}-\left(\sigma-\frac{1}{r+1}\right) x_{i} z_{i}-2 \sigma^{2} y_{0}+\sigma x_{0} z_{0} \\
& \dot{y}_{i}=r x_{i}-y_{i}-2 \sigma r\left(1+\frac{1}{\sigma(r+1)}\right) y_{i}-\left(1+\frac{1}{r+1}\right) x_{i} z_{i}+2 \sigma r y_{0}-x_{0} z_{0} \\
& \dot{z}_{i}=-d z_{i}+\left(d+\frac{1}{d}\right) x_{i} y_{i}-d x_{0} y_{0} \\
& \forall i=1,2,3
\end{aligned}
$$

In this case the non-central nodes are not always synchronize but it we choose

$$
\Lambda_{i}=\mu_{i}(i=1,2,3)=A^{-1}=\left(\begin{array}{ccc}
-\frac{1}{\sigma(r+1)} & -\frac{1}{r+1} & 0 \\
-\frac{r}{\sigma(r+1)} & \frac{1}{r+1} & 0 \\
0 & 0 & \frac{1}{d}
\end{array}\right)
$$

Then the dynamics of non-central nodes are given by

$$
\begin{aligned}
& \dot{x}_{i}=-\sigma\left(x_{i}+y_{i}\right)-\frac{2}{r+1} y_{0}+\frac{1}{r+1} x_{0} z_{0} \\
& \dot{y}_{i}=r x_{i}-y_{i}-\frac{2 r}{r+1} y_{0}-\frac{1}{r+1} x_{0} z_{0} \\
& \dot{z}_{i}=-d z_{i}+\frac{1}{d} x_{0} y_{0} \\
& \forall i=1,2,3
\end{aligned}
$$

Hence by Theorem 3.1. the non-central nodes are synchronized clearly, are shown in Fig.7(a)-Fig.7(c).

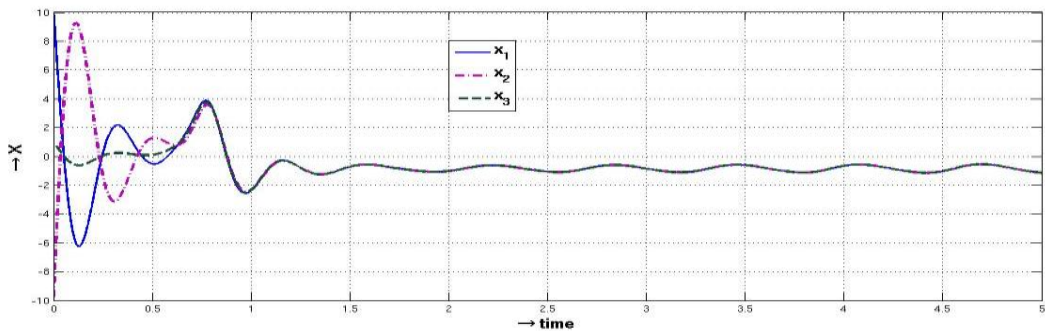

Fig.7(a). Time evolution of $x_{1}, x_{2}$ and $x_{3}$ when $\lambda=\mu$ for simulation 3 . 


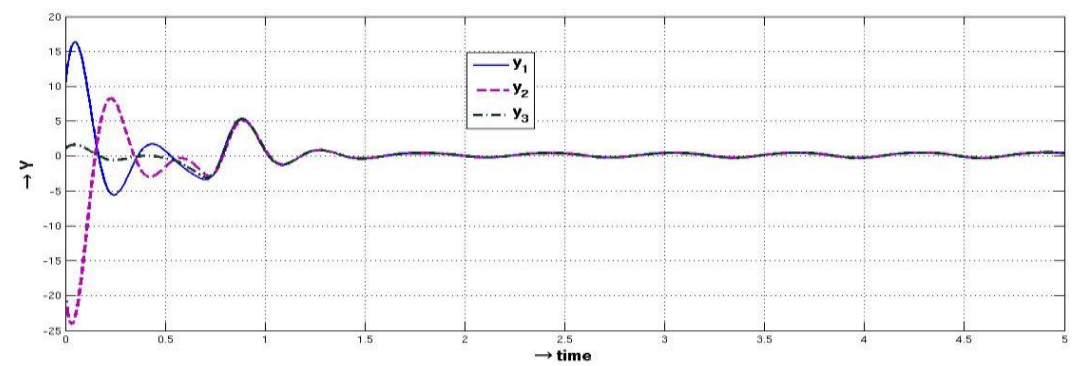

Fig.7(b): Time evolution of $y_{1}, y_{2}$ and $y_{3}$ when $\lambda=\mu$ for simulation 3 .

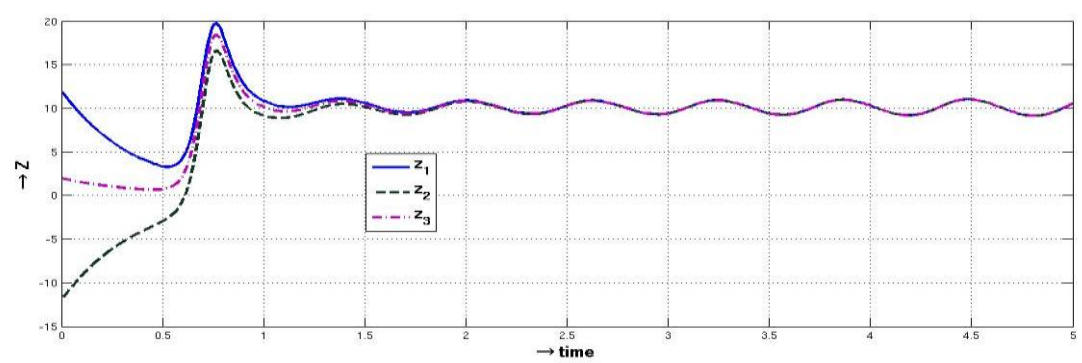

Fig.7(c): Time evolution of $z_{1}, z_{2}$ and $z_{3}$ when $\lambda=\mu$ for simulation 3 .

\section{Conclusions}

We have nicely generalizes the results of Tang et.al.[22] on chaos synchronization of star network. We derive the sufficient condition for generalized synchronization in a star network. We have tested our conditions by numerical simulations and observe nice agreement with the analytical conditions. Our scheme is applicable for all chaotic systems i.e. our scheme is independent of chaotic systems. After generalized synchronization, the functional relationship among the state variables of the non-central nodes are known in advance in our method. Therfore we have complete control of designing the dynamics of the state variables of the non-central nodes. Our design of dynamics of the individual nodes in star network for generalized synchronization may have potential applications in secure communication, information processes and neurosciences.

\section{References}

[1] L.M. Pecora and T.L. Carroll, Synchronization in chaotic systems, Phys. Rev. Lett, 64 (1990) 821-824.

[2] S.Tang and JM.Liu, Experimental verification of anticipated and retarded synchronization in chaotic semiconductor lasers, Phys.Rev.Lett, 90(2003) 194101. 
[3] N.F.Rulkov, M.M.Suschik and L.S.Tsimring, Generalized synchronization of chaos in directionally coupled chaotic systems, Phys.Rev.E, 51(1995) 980994.

[4] ZM. Ge and CC. Chen, Phase synchronization of coupled chaotic multiple time scales systems, Chaos, Soliton \& Fractals, 20(2004) 639-647.

[5] Li. Chuangdong, Coexistence of anti-phase and complete synchronization in coupled Chen system via a single variable, Chaos, Soliton \& Fractals, 38(2008) 462-464.

[6] Erjaee,G.H., On analytical justification of phase synchronization in different chaotic systems, Chaos, Soliton \& Fractals, 3(2009) 1195-1202.

[7] Li. C, Liao X and Wong K, Lag synchronization of hyperchaos with application to secure communications, Chaos, Soliton \& Fractals, 23(2005) 183-193.

[8] A Tarai(Poria), S Poria and P. Chatterjee, Synchronization of bidirectionally coupled chaotic Chen's system with delay, Chaos Solitons and Fractals, 41 (2009) 643-647.

[9] S.Poria, A.Tarai and P.Chatterjee, Synchronization threshold of a coupled ndimensional time-delay system, Chaos, Soliton \& Fractals, 41(2009) 11231124.

[10] Park JH, Adaptive synchronization of hyperchaos Chen system with uncertain parameters, Chaos, Soliton \& Fractals, 26(2005) 959-964.

[11] Hilaire Fotsin, Samuel Bowong and Jamal Daafouz, Adaptive synchronization of two chaotic systems consisting of modified Vander PolDuffing and Chua oscillators, Chaos, Soliton \& Fractals, 26(2005) 215-229.

[12] Ge. Zheng-Ming , Adaptive synchronization of unidirectional and mutual coupled chaotic systems, Chaos, Soliton \& Fractals, 26(2005) 882-888.

[13] Yassen, M.T., Adaptive synchronization of Rossler and Lu systems with fully uncertain parameters, Chaos, Soliton \& Fractals, 23(2005) 1527-1536.

[14] Chen, Heng-Hui, Adaptive synchronization of linearly coupled unified chaotic systems, Chaos, Soliton \& Fractals, 40(2009) 598-606.

[15] Li, Guo-Hui, An observer-based anti-synchronization, Chaos, Soliton \& Fractals, 29(2006) 495-498.

[16] El-Dessoky, Synchronization and anti-synchronization of hyperchaotic Chen system, Chaos, Soliton \& Fractals, 39 (2009) 1790-1797.

[17] Li, Guo-Hui and Zhou Shi-Ping, Anti-synchronization in different chaotic systems, Chaos, Soliton \& Fractals, 32(2007) 516-520.

[18] M.A.Khan, S.N.Pal and S.Poria, Generalized anti-synchronization of different chaotic systems, Int. J. of Applied Mechanics and Engineering, 17(2012) 83-89.

[19] J.Hu, S.Chen and L.Chen, Adaptive control for anti-synchronization of Chua's chaotic system, Physics Letters A, 339(2005) 455-460.

[20] D.J. Watts and S.H.Strogatz, Collective dynamics of 'small-world' networks, Nature, 393(1998) 440-442. 
[21] R.Albert, H.Jeong and A.L.Barabasi, Emergence of scaling in random networks, Science, 286(1999)509-512.

[22] L Tang, Z Kang, Research of network synchronization based on three dimensional chaotic system, IEEE Proc. of IC-NIDC2009. 